\title{
Inputs and Outputs of the Intelligence Cycle: a highway to shared definitions and a knowledge base.
}

\author{
Gonçalo João \\ Universidade Autónoma de Lisboa \\ Departamento de Ciências Económicas e Empresariais
}

September 9, 2019

\begin{abstract}
The establishment of a common ground for basic definitions and tools of competitive intelligence to practitioners and academics can be partially achieved by fully recognize and comprehend the inputs and outputs of the intelligence cycle. Nevertheless there are some additional issues to address in order to reach the so looked-for shared definitions and knowledge base.
\end{abstract}

\section{Introduction}

My first contact with competitive intelligence was in 2003 during a post-graduate course on Information Systems and Technology. However, it was only later in 2009, when I began thinking of a theme for my $\mathrm{PhD}$ thesis that I began collecting and reading every book, paper and article about competitive intelligence I could get my hands on. Since then, a kind of theory has grown within me. I often find myself discussing competitive intelligence issues with colleagues and friends. I started to see competitive intelligence in everyday aspects of my life and in the daily news that I followed on the media. Soon, I found myself fighting against those who do not 
distinguish competitive intelligence from industrial espionage, and discussing with those who do not value competitive intelligence and rather discussed business or marketing intelligence. Although I have always defended competitive intelligence based on all I have learned from books, papers and articles, I do realize that competitive intelligence does not meant the same to everyone, nor to everyone author I have read. As Jonathan Calof (2008) stated "We lack shared definitions and a consistent knowledge base". He wrote it when addressing the issue of the professionalization of competitive intelligence. Regarding this particular item, he also stated that there are some inconsistencies found in presentations and articles about competitive intelligence. I too have found several ambiguous and inaccurate definitions and terms used by both professionals and academics. Most of them are, in my opinion, simple misinterpretations of the intelligence cycle, and are also a product of different definitions of competitive intelligence.

It is my strong belief that one possible solution could be found in the intelligence cycle. Understanding the inputs and outputs of the intelligence cycle might help both professionals and academics to talk and write about competitive intelligence in a more consistent and accurate manner, thus contributing to shared definitions and a knowledge base that the profession so lacks. This article does not intend to tell competitive intelligence professionals and practitioners how to conduct their jobs nor to adjust studies and manuscripts of academics and researchers. Its purpose is not to build a single definition of competitive intelligence either, nor a common accepted intelligence cycle to the community. This article is an alert, a wake up call if you must, to the inputs and outputs of the intelligence cycle, and how they can help understand better the terms around competitive intelligence. Only then will it be possible to think about a common definition of competitive intelligence and a common intelligence cycle.

\section{The Intelligence Cycle: Inputs and Outputs}

For the purpose of this article allow me to consider the commonly referred as the classic four steps intelligence cycle. This intelligence cycle follows planning and direction, collection, analysis, and dissemination (see Figure 1), as defined by Kahaner (1996). 


\section{Planning and Direction}

The planning and direction step is a process that identifies the needs of the competitive intelligence customer, establishes a collection and analysis plan, and keeps the customer informed (Kahaner, 1996). The customers can be senior management or other decision-makers, management processes or procedures, such as budget or strategic programs, and the competitive intelligence function (Herring, 2005). A more proactive planning and direction could mean an iterative and on-going process (Kindler, 2003).

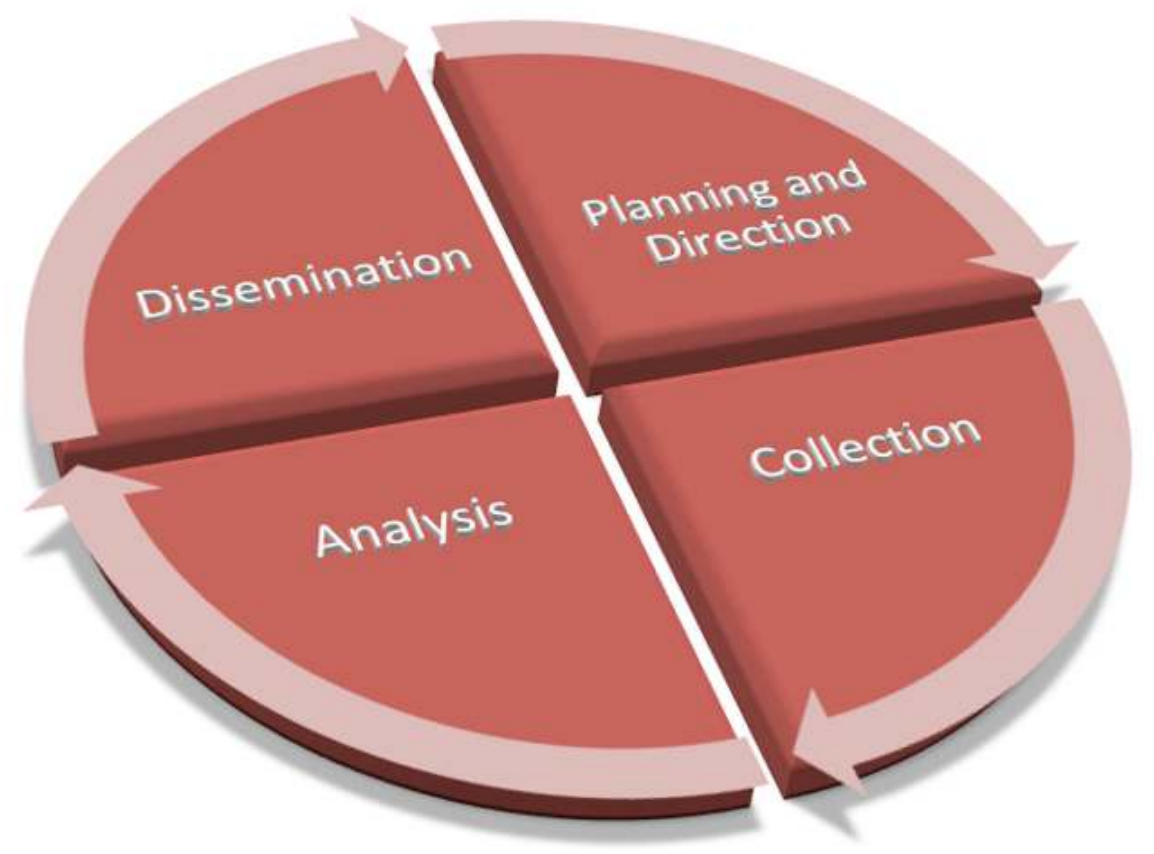

Figure 1 - The Intelligence Cycle adapted from Kahaner (1996)

The main input of this first step is the needs of the competitive intelligence customers, or key intelligence needs (Ferreyra, 2004), which are often identified using the key intelligence questions and later translated into key intelligence topics. Do not confuse key intelligence questions with key intelligence topics, the first is a subordinate of the last (Herring, 1999) and a tool to identify key intelligence topics. A key intelligence 
topic should be related to a decision and/or action, and should have a timeline (Taborda \& Ferreira, 2002). There are three types of key intelligence topics: strategic and tactical decisions, early-warning topics, and descriptions of key players (Johnson, 2004). The key players could be competitors, suppliers, and customers of the organization; new entrants and substitute products from the markets where the organization operates; or any other players from the political, economical, social, technological, ecological, and legal environment of the organization. The players here listed are taken from two important models when analyzing the competitive environment of the organization, the five forces model of Porter (1980) and the macro-level PESTEL analysis (Hedin, Vaarnas \& Vanhala, 2007). Do not limit your collection or analysis to these two models. They are a good starting point, but depending on the industry in analysis, more specific models and frameworks should be applied. Some authors often referred to the needs of the competitive intelligence customers simply as intelligence needs. Although everyone in the profession quickly understands it, to best establish shared definitions and notions and to allow an easily recognition by the scientific community, authors should refer to the complete concept: needs of the competitive intelligence customers, or any accepted acronym.

The output of the planning and direction step is a plan for timely collection and analysis of information related to the issue at hand. If the key intelligence topics are well produced, leading to a decision and/or action, including a list of sources of information, a list of proper analytical tools, and a timeline, then it is acceptable to say that the output of planning and direction is the key intelligence topic itself, as a plan.

\section{Collection}

The second step of the intelligence cycle is the gathering of information in a legal and ethical manner, and its processing and storing in a form that allow further manipulation to be analyzed in the next step (Kahaner, 1996).

Apart from the plan produced in the previous step, expressed in the key intelligence topics, the main input of collection is information. From a competitive intelligence perspective, information can be found in primary or secondary, and in internal or external sources. Nevertheless, the input is always information. Primary sources of information are those where the information is in its raw state, unchanged (Kahaner, 
1996) and can be often collected through human contact and observation (Taborda \& Ferreira, 2002). Secondary sources of information are those who have been previously selected from larger sources or altered by opinion (Kahaner, 1996) and can be found in published sources (Taborda \& Ferreira, 2002). Internal sources of information are reports, documents and databases of the organization and its employees, also known as the intellectual capital of the organization. Depending on the issue or issues at hand, external sources of information can be any of the key players identified before, which can be contacted and the information collected through personal contact, observation, the internet, the media, expert networks, in conferences and trade shows, in government and industry reports, just to mention a few.

This step cannot be addressed without referring to the legal and ethical aspects of collection itself. Even if the purpose of this article is not to discuss the legal and ethic codes of collection, it is always adequate to mention a few sources of ethical codes or important laws to the information collector: the Code of Ethics of SCIP, the Ten Commandments of Legal and Ethical Intelligence Gathering of Fuld \& Company, the U.S. Economic Espionage Act, the U.S. Uniform Trade Secrets Act, the European Data Protection Directive, the U.K. Data Protection Act, local and government patent and intellectual property agencies, and current and past non-disclosure agreements signed by the primary sources of information contacted. A successful competitive intelligence function, and the organization itself for that matter, should have a code of ethics, well known and understood by everyone in the organization (Kindler, 2003). This code of ethics should not be restricted to the collection step, but applied to all steps in the intelligence cycle and all aspects of the competitive intelligence function, as explained by John Prescott in his article "CI and Ethics: either you have it or you don't" (Prescott, 2003). And in case of doubt, remember the rule thumb of Karen Rothwell (2003): "When uncertain, refer all your questions regarding legal and ethical intelligence practices to your company Legal Department”.

Common inaccurate statements in this step are often, as some authors refer to the gathering or collection of intelligence. Intelligence cannot be gathered nor collected. However, it can be purchased, if necessary, through outsourcing. In any case, intelligence is produced through a process known as the intelligence cycle. Actions of 
the collection step of the intelligence cycle should be referred as collection, process and codification of information.

Consequently, the output of the collection step is collected, processed and coded information. Although it is not mandatory the storage of the information collected in an electronic format, nowadays, is common to use a competitive intelligence system to store that information. One of the advantages of such a system is the easy access to the information in a decentralized organization or competitive intelligence function.

\section{Analysis}

The third step of the intelligence cycle is analysis, where the information collected is transformed into intelligence (Bernhardt, 1994; Kahaner, 1996). Some authors state that this step is the most difficult of all of the steps of the intelligence cycle. Basically, the analyst looks for trends and patterns in the information collected (Kahaner, 1996; Taborda \& Ferreira, 2002) and fills the blanks with educated guesses (Kahaner, 1996), creative analysis or alternative thinking (Calof, 1998).

There are several models and frameworks to achieve this goal in analysis, but essentially, the analyst looks into the several pieces of information collected with the key intelligence topic in mind, adds judgments and interpretative capabilities, relates them while providing a grounded explanation, identifies its implication to the organization, and establishes strategic options and action recommendations (Sawka, 2002). The analyst must be persistent, rigorous and deep in its analysis, should not discard common sense, and beware of the timeline of the key intelligence topic in analysis and the moment of change (Taborda \& Ferreira, 2002). Moment of change is a concept introduced by Leonard Fuld that represents a massive change in the environment where a load of stories are produced in the media, a large quantity of paperwork is filled and many public-domain documents are produced (Kahaner, 1996). The hardest judgment here is in choosing the right analytical tools to the specific issue in analysis. The five forces model of Porter (1980) could help identify the main forces in the industry, but should be complemented with a more deeply analysis, either on the competition topic, or on all the other four. In order to do that a financial and SWOT analysis, competitor and key decision-makers profiling, leadership and patent analysis on the major competitors is imperative. This option is 
highly dependent on the time and resources available. To select the right analytical tools for each key intelligence topic, analysts could use the rating system FAROUT based on six common characteristics of each tool. The system rates the analytical tool based on its future orientation, accuracy, resource-efficiency, objectivity, usefulness and time (Fleisher \& Bensoussan, 2000).

This is the step where I have found fewer inaccuracies, nevertheless, allow me to recapitulate some terms. In analysis, competitive intelligence analysts and practitioners analyze the collected, processed and coded information in the previous step - collection - and look for trends and patterns. They use their own personnel judgments and interpretative capabilities, along with analytical tools. These analytical tools can be found in the general management discipline and its sub-disciplines, such as strategic management, finance, marketing, knowledge management, product management and development, technological innovation, among others. The result of the analysis is intelligence, which in this context can be defined as strategic options and action recommendations for the decision-makers, and is also the single output of the analysis step of the intelligence cycle. Other definitions refer intelligence as actionable recommendations and actionable insights on the environment. Professionals and academics should not use the term information when defining intelligence; it only confuses those who have not worked with competitive intelligence before, or are studying and trying to understand competitive intelligence. However, when using information to define intelligence, just add some adjectives in the term information, like Kahaner (2002) did: "Intelligence (...) is a collection of information pieces that have been filtered, distilled, and analyzed". When considering the hierarchy of knowledge terminology, which goes from raw data to knowledge, if one chooses to use one of its terms, opt for one closer to knowledge than to information. As Kahaner (2002) so well put it: "Another term for intelligence is knowledge".

\section{Dissemination}

Finally, the last step of the intelligence cycle is dissemination. Dissemination is the distribution or delivery of intelligence to the customers of the competitive intelligence or to who requested it (Bernhardt, 1994; Kahaner, 1996). There are several ways to disseminate intelligence that goes from newsletters, intelligence reports to 
presentations and meetings with the customers. The competitive intelligence team or practitioner should be prepared to defend their recommendations or actions with logical arguments (Kahaner, 1996), using presentations and persuasive skills, and appropriate format or media to each case (Calof, 1998).

The input of this step is intelligence and the output is any form or product the competitive intelligence practitioner chooses to use. Those products are often referred as newsletters, distributed to a large part of the organization, intelligence reports to decision-makers and mid-level managers, and presentations usually to top management and high-level decision-makers. No matter what kind of intelligence reports competitive intelligence practitioner preferred to use for each specific cases, the contents of any of them should be a timely actionable intelligence.

Fortunately, most of what has been written about dissemination, has been done correctly from an accuracy and consistency point of view, for they focus on the best practices on delivering intelligence. Nonetheless, some authors wrongly refer to the delivery of the analysis results or even the analysis itself. Dissemination is not about the delivery of the analysis, but the delivery of intelligence, produced and created from the analysis results combining with strategic options and action recommendations. The results of analysis are unlikely to include actions, they have to be included as the practitioners interpreted them in the light of other analysis conducted and their own convictions. Deliver intelligence, not analysis results. Do not deliver a SWOT matrix; deliver recommendations based on the SWOT analysis conducted, and eventual future threats and opportunities.

\section{Closing the Cycle}

When the intelligence cycle reaches the final step another cycle begins. Usually, the intelligence is delivered and the competitive intelligence function goes up to the next key intelligence topic at hand. However, sometimes when delivering intelligence to the competitive intelligence customers, those might require new needs or come up with new questions related to the intelligence just delivered, thus starting a new cycle. On the other hand, sometimes while preparing the intelligence dissemination, or even, before that, while conducting analysis, competitive intelligence practitioner identifies 
new needs of competitive intelligence customers, giving origin to a new cycle, where the input of the planning and direction step is not the customer itself. And thus, also creating a new output from the dissemination step (see Figure 2).

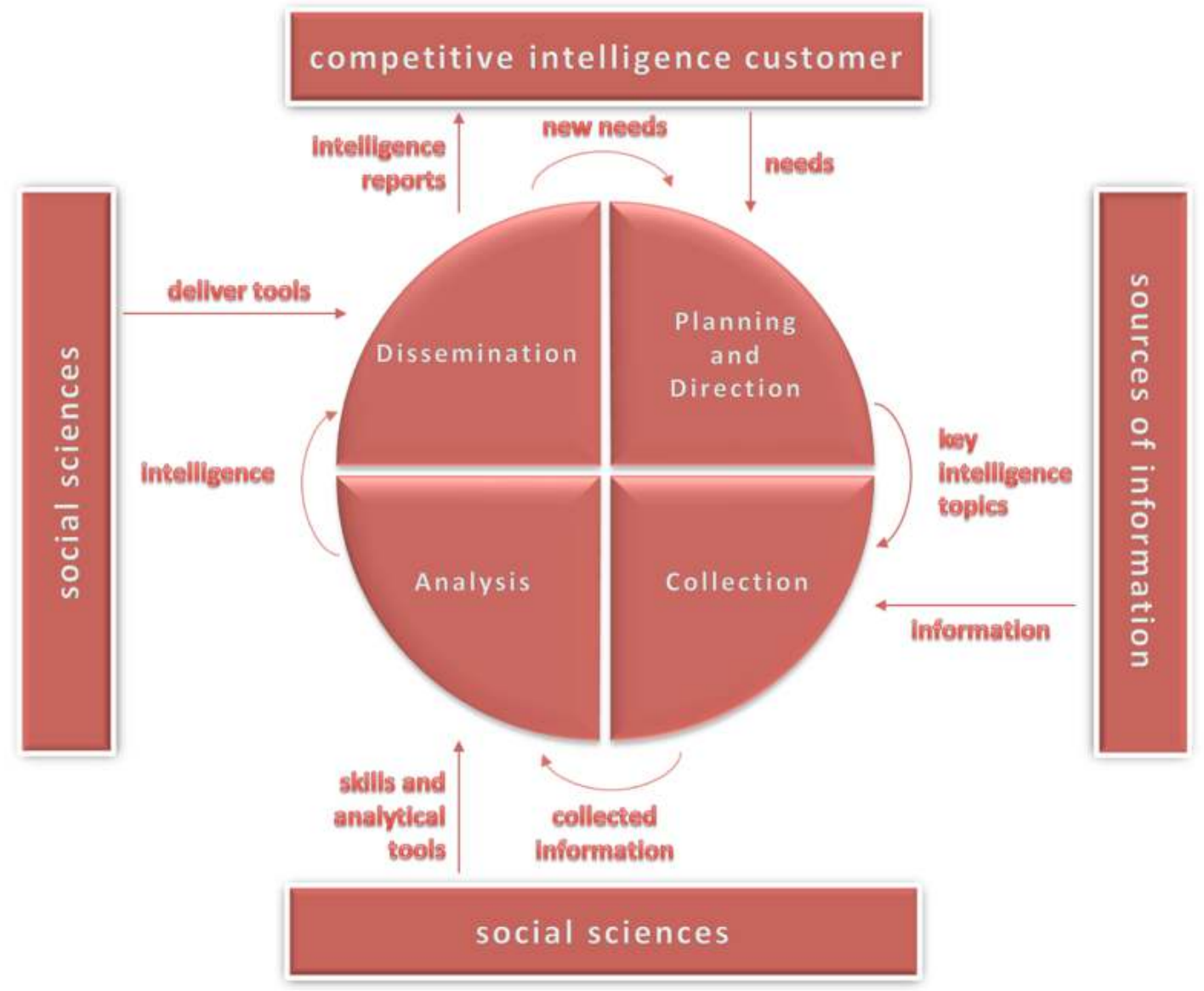

Figure 2 - The Inputs and Outputs of the Intelligence Cycle

\section{Additional Steps}

There are additional steps in other intelligence cycles found in articles of academic journals and magazines. The most common are the processing, production, feedback and management steps. The first three, are steps that are either implicit in the classic four steps cycle or in the competitive intelligence function itself. The processing step is included in the collection step of our intelligence cycle. The production step is included in the analyze step when intelligence is produced. The feedback step is implicit in the dissemination step, although sometimes it could be hard to get feedback from the competitive intelligence customers. A different step is the management step identified by some authors. Its focus is the management of the 
intelligence cycle itself. For the purpose of the inputs and outputs of the intelligence cycle, the management step bring less worries, as I have never read about managing intelligence instead of managing the intelligence cycle. On the other hand, processing intelligence, producing information or getting feedback from delivered information, are unfortunately more used terms. Once more, information is collected, processed, stored, filtered, distilled and analyzed. Intelligence is produced and disseminated or delivered.

\section{Competitive Intelligence: Guidelines to a Definition}

Most of the definitions of competitive intelligence I come across have five things in common: the systematic, legal and ethical characteristics, a classification, and references to the processes utilized. Stating that competitive intelligence is a systematic, legal and ethical process, it is another step towards shared definitions and a knowledge base for the professionalization of competitive intelligence, and also to the building of the theory. Even the discussion about competitive intelligence classification, either as being a process, a product, both of them, or a program, contributes positively to that purpose. However, when defining competitive intelligence, and exemplifying the process steps with information, does not contribute at all. When practitioners and academics refer to competitive intelligence as the gathering, analysis and dissemination of information, or distribution and acting upon information, the idea remains that competitive intelligence is just collecting and disseminating information. Once more, competitive intelligence collects information, but delivers intelligence, which then the organization can act upon. When defining competitive intelligence, authors that feel the urge to include the steps of the intelligence cycle, must remember that. Or alternatively, refer to the intelligence cycle as the process used, and then define it. One of the simplest definitions ever is "competitive intelligence is a tool for the decision-maker" (Taborda \& Ferreira, 2002). After which the authors define the intelligence cycle, their inputs and outputs, and their implications. The definition of competitive intelligence should refer or include the following aspects: systematic process or program, legal and ethic, gathering or collection and analysis of information, dissemination or delivery of intelligence, decision-makers or decision-making, and if necessary, actionable intelligence, insights or foresight, intelligence that allows the organization act upon, 
competitive advantages, competitive or business environment, general business trends, threats and opportunities, and long term focus.

\section{Conclusion}

Understanding the inputs and outputs of the intelligence cycle in order to conduct systematic, legal and ethical competitive intelligence, might help both practitioners and academics to establish a common ground when addressing its definitions, tools, and implications. Converging to shared definitions and a common knowledge base will contribute to the professionalization of competitive intelligence, but also to a more likely acceptance among academics and organizations. It is common sense, that competitive intelligence is performed in organizations; it is our responsibility, both academics and practitioners, to ensure that it is performed correctly, legally and ethically. We must talk, write and breathe the right terms, the right definitions, the right tools, and the right competitive intelligence. Otherwise, we loose all logical arguments to defend competitive intelligence against industrial espionage, business intelligence, marketing intelligence, and so many new disciplines and academic trends surrounding decision-making processes and actionable intelligence.

Nevertheless, let's not forget the main reason of this article, the article of Jonathan Calof (2003). There are other issues to consider: the lack of understanding of competitive intelligence by managers and employees; the lack of importance of competitive intelligence function by managers, the lack of educational credibility of competitive intelligence compared with others disciplines, and the lack of recognized academic scholarship due to the almost inexistence academic research and publication on competitive intelligence (Calof, 2008). Maybe someone will address them soon, and provide us with a solution or their opinions.

\section{References}

Bernhardt, D. C. (1994). "I want it fast, factual, actionable" - Tailoring competitive intelligence to executives' needs. Long Range Planning, 27(1), 12-24.

Calof, J. (1998). Increasing your CIQ: the competitive intelligence edge. Economic Development Journal. Available from www.ecdevjournal.com, last accessed on May $17^{\text {th }}, 2010$. 
Calof, J. (2008). Competitive intelligence: are we really becoming a profession? Competitive Intelligence Magazine, 11(5), 16-20.

Ferreyra, J. C. (2004). Pharmaceutical CI planning: a seven matrix methodology. Competitive Intelligence Magazine, 7(5), 40-43.

Fleisher, C. \& Bensoussan, B. (2000). A FAROUT way to manage CI analysis. Competitive Intelligence Magazine, 3(2), 37-40.

Hedin, H., Vaarnas, M. \& Vanhala, V. (2007). Market intelligence for the strategy and planning process. Competitive Intelligence Magazine, 10(6), 25-30.

Herring, J. P. (1999). Key intelligence topics: a process to identify and define intelligence needs. Competitive Intelligence Review, 10(2), 4-14.

Herring, J. P. (2005). Create an intelligence program for current and future business needs. Competitive Intelligence Magazine, 8(5), 20-27.

Johnson, S. (2004). Planned decisions and pending actions. Competitive Intelligence Magazine, 7(6), 43-45.

Kahaner, L. (1996). Competitive intelligence - how to gather, analyze, and use information to move your business to the top. Simon \& Schuster, New York.

Kindler, T. (2003). Creating a successful CI operation in today's corporate environment. Competitive Intelligence Magazine, 6(5), 6-9.

Porter, M. E. (1980). Competitive strategy: techniques for analyzing industries and competitors. Free Press, New York.

Prescott, J. (2006). CI and ethics: either you have it or you don't. Competitive Intelligence Magazine, 9(2), 6-10.

Rothwell, K. (2008). Ethics: the limits of intelligence gathering. Competitive Intelligence Magazine, 11(2), 34-35.

Sawka, K. (2002). Stages of Analysis. Competitive Intelligence Magazine, 5(6), 4647.

Taborda, J. \& Ferreira, M. (2002). Competitive intelligence - conceitos, práticas e beneficios. Pergaminho, Cascais. 